\title{
Atención Plena: Validación del Five Facet Mindfulness Questionnaire (FFMQ) en estudiantes universitarios chilenos
}

\author{
Mindfulness: Validation of the Five Facet Mindfulness Questionnaire (FFMQ) in Chilean \\ University Students
}

\author{
Carlos Schmidt \\ Eugenia V. Vinet \\ Universidad de La Frontera, Chile.
}

(Rec: 24 mayo 2013 / Acept: 25 marzo 2015)

\begin{abstract}
Resumen
Este estudio tuvo por objetivo determinar las propiedades psicométricas del Five Facet Mindfulness Questionnaire (FFMQ) en estudiantes universitarios de la ciudad de Temuco en Chile $(n=399)$. El FFMQ es una prueba de autoreporte compuesta por 39 ítems, que mide la tendencia general a proceder con Atención Plena a partir de cinco habilidades: Observación, Descripción, Actuar con Conciencia, Ausencia de Juicio y Ausencia de Reactividad. El diseño es descriptivo correlacional, de grupo único. Los resultados muestran una confiabilidad adecuada y la asociación de Atención Plena con dimensiones de personalidad del Modelo de los Cinco Factores de Costa y McCrae, con la Autoactualización de las teorías humanistas y una relación inversa con estados emocionales negativos de depresión, ansiedad y estrés. Los análisis muestran que la versión nacional del FFMQ posee adecuadas propiedades psicométricas para ser utilizado en estudiantes universitarios chilenos y permite discriminar entre meditadores y no meditadores.

Palabras clave: Atención Plena, meditación, personalidad, sintomatología, autoactualización
\end{abstract}

\begin{abstract}
This study aimed to determine the psychometric properties of the Five Facet Mindfulness Questionnaire (FFMQ) in university students from the city of Temuco, Chile $(n=399)$. The FFMQ is a self-report test consisting of 39 items, which measures the general tendency to proceed with Mindfulness by using five skills: Observation, Description, Acting with Awareness, Non-judging of inner experience and Non-reactivity to inner experience. The design is descriptive correlational of unique group. The results show adequate reliability and Mindfulness association with personality dimensions of the Five Factor Model of Costa and McCrae, with the Self-Actualization of humanistic theories and an inverse relation to negative emotional states of depression, anxiety and stress. Analyzes show that the Chilean version of FFMQ has adequate psychometric properties for use in Chilean university students and discriminates between meditators and non-meditators. Keywords: Mindfulness, meditation, personality, symptomatology, self-actualization
\end{abstract}

Correspondencia: Correspondencia sobre este estudio debe dirigirse a Carlos Schmidt, email:vicenteschmidt@gmail.com

Nota: Este estudio fue financiado por el Proyecto DI12-2021 de la Dirección de Investigación de la Universidad de La Frontera. 


\section{Introducción}

La Atención Plena, traducción del término en inglés Mindfulness (Miró, 2007), se puede comprender como una capacidad inherente a la conciencia humana que permite atender a los fenómenos que se experimentan en cada momento presente, de un modo en el que se aceptan tal y como emergen en la conciencia, sin realizar juicios sobre ellos (Baer, Smith y Allen, 2004; Brown y Ryan, 2003). Asimismo, la Atención Plena supone dirigir la atención al momento presente que estamos viviendo, con una actitud de compasión, interés, apertura y amabilidad, independientemente de si la experiencia resulta agradable o desagradable (Kabat-Zinn, 2003). Así, la práctica de Atención Plena significa entonces cultivar una actitud consciente hacia todo tipo de experiencias y procesos mentales (Jankowski y Holas, 2014).

La Atención Plena tiene un fuerte parentesco con las prácticas de la cultura oriental adscritas al Budismo Zen, que se remontan por más de 2.500 años y sugieren que mayores niveles de Atención Plena se pueden desarrollar a partir de la práctica regular de meditación (Baer et al., 2004; Kabat-Zinn, 2003). En la última década, los estudios han enfatizado en los beneficios para la salud mental, relacionados con el desarrollo de la Atención Plena en el día a día (Baer et al., 2008; Brown y Ryan, 2003; Carlson y Brown, 2005). Estos hallazgos han alimentado el interés de la psicología occidental por seguir estudiando la naturaleza del constructo y por incorporar diversas técnicas y métodos que desarrollen la Atención Plena en sus intervenciones clínicas (Baer, Smith, Hopkins, Krietemeyer y Toney 2006; Vallejo, 2006). Es por esto que la práctica tradicional de Atención Plena se ha adaptado e incorporado para su uso en varias intervenciones terapéuticas, como son la Terapia Conductual Dialéctica (Linehan, 1993a, 1993b), el Programa Reducción de Estrés Basado en Mindfulness (Kabat-Zinn, 1982, 1990) y la Terapia Cognitiva basada en la Atención Plena (Segal, Williams y Teasdale, 2002). Estas intervenciones conceptualizan la Atención Plena como un conjunto de habilidades que pueden ser aprendidas y practicadas con el fin de reducir los síntomas psicológicos y mejorar la salud y el bienestar (Baer et al., 2006). Específicamente, las investigaciones han demostrado que las intervenciones basadas en Atención Plena logran una disminución de la rumiación (Chambers, Lo y Allen, 2008), reducción en la reactividad (Cahn y Polich, 2009), la ansiedad y los síntomas depresivos (Hoffman, Sawyer, Witt, y Oh, 2010).
Baer et al. (2006) examinaron la estructura de cinco cuestionarios que se han desarrollado sobre la Atención Plena, evaluando su coherencia interna y la correlación entre ellos. Estos son: el Mindful Attention Awareness Scale (MAAS; Brown y Ryan, 2003; Carlson y Brown, 2005), el Freiburg Mindfulness Inventory (FMI; Buchheld, Grossman y Walach, 2001), el Kentucky Inventory of Mindfulness Skills (KIMS; Baer, Smith y Allen, 2004), la Cognitive and Affective Mindfulness Scale (CAMS; Feldman, Hayes, Kumar y Greeson, 2004) y el Mindfulness Questionnaire (MQ; Chadwick, Hember, Mead, Lilley, y Dagnan, 2005). El análisis factorial exploratorio de estos cuestionarios sugirió cinco facetas claras de la Atención Plena y consolidó un nuevo cuestionario denominado Five Facet Mindfulness Questionnaire (FFMQ, Baer et al., 2006), que mide la tendencia general a proceder con Atención Plena a partir de cinco factores: (1) Observación; incluye darse cuenta o asistir a experiencias internas y externas, tales como sensaciones, cogniciones, emociones, sonidos y olores. (2) Describir; se refiere a etiquetar experiencias internas con las palabras. (3) Actuar con conciencia; incluye la asistencia a las propias actividades del momento y puede ser contrastado con comportarse mecánicamente mientras la atención se centra en otro lugar (piloto automático). (4) No hacer juicio de la experiencia interna; se refiere a adoptar una postura no evaluativa hacia pensamientos y sentimientos. (5) No reaccionar ante la experiencia interna; es la tendencia de permitir que los pensamientos y sentimientos fluyan, sin quedar atrapados en o dejarse llevar por ellos. Baer et al. (2008) reportan buenas propiedades psicométricas del FFMQ.

Teóricamente, la Atención Plena se ha descrito como un estado y como rasgo (Brown y Ryan, 2003; Segal et al., 2002), pero también como una habilidad que se puede desarrollar con la práctica (Bishop et al., 2004; Linehan, 1993b). Al estudiar su relación con el Modelo de los Cinco Factores (MCF; Costa y McCrae, 1992), se ha observado que la práctica de la Atención Plena se asocia con la reducción del afecto negativo característico del Neuroticismo, y con la receptividad a los sentimientos y el interés por los estímulos ambientales, coherente con la Apertura a la Experiencia (Baer et al., 2006). Sobre la relación con estados emocionales negativos, Baer et al. (2006) demostraron que las facetas Actuar con Conciencia, No Juzgar y No reaccionar ante la experiencia, tienen validez incremental en la predicción de síntomas psicológicos, asociándose la Atención Plena con la reducción de sintomatología (Baer et al., 2008). La Atención Plena también ha mostrado congruencia teórica con los planteamientos de las terapias humanistas, encontrándose 
similitud con las características que la psicología occidental atribuye a las personas auto-actualizadas. Éstas son personas que viven en el presente con plena conciencia y en contacto con la realidad, además son individuos orientados hacia sí mismos e independientes (Shostrom, 1977), estas personas serían quienes pueden actuar más fácilmente con Atención Plena, es decir, con aceptación y conciencia plena del momento presente.

En Chile ha surgido interés por estudiar, comprender y trabajar con este constructo. Al respecto, Irarrázaval (2010) la describe como una forma de observar y conocer la naturaleza de la propia mente y el modo en que cada cual concibe su realidad. Por otro lado, Medeiros y Pulido (2011) evaluaron la aplicabilidad y eficacia del programa de reducción de estrés basado en la Atención Plena diseñado por Kabat-Zinn $(1982,1990)$ en funcionarias de un hospital de la ciudad de Santiago de Chile. Finalmente, existe en la actualidad una versión en español del FFMQ, desarrollada en Chile por Cárcamo y Cruz-Coke (2009); sin embargo, esta versión no cuenta con propiedades psicométricas conocidas en nuestro país, siendo deseable su validación tanto para el estudio del constructo y su relación con otros conceptos psicológicos, como para la evaluación de intervenciones que consideren la práctica de la Atención Plena y los beneficios que trae para las personas.

El propósito de la presente investigación es estudiar, en una muestra chilena, las propiedades psicométricas del FFMQ con el objeto de proporcionar a los investigadores chilenos un instrumento idóneo para trabajar con el constructo de Atención Plena en el desarrollo de futuros estudios e intervenciones. Como objetivos específicos se pretende: (1) Determinar la confiabilidad del instrumento a través de su consistencia interna, y (2) Determinar su validez convergente y discriminante a través de su asociación con otros constructos relacionados teóricamente de acuerdo a los antecedentes señalados previamente.

Como hipótesis, sustentadas en la literatura internacional y dado que se cuenta con medidas apropiadas en el contexto sociocultural chileno, se espera que la Atención Plena se relacione de modo específico con los siguientes constructos: 1. Con las siguientes dimensiones del MCF: (a) Relación inversa con Neuroticismo, (b) Relación directa con Apertura a la experiencia y (c) Relación no significativa con Extroversión.

2. Relación inversa con estados emocionales negativos de depresión, ansiedad y estrés.

3. Relación directa con la Tendencia Autoactualizante de las terapias humanistas.

\section{Método}

\section{Participantes}

La muestra, seleccionada por un muestreo no probabilístico, por conveniencia, estuvo integrada por 399 estudiantes universitarios de la ciudad de Temuco, de ellos 166 corresponden a la Facultad de Medicina y 104 a la Facultad de Ingeniería de la Universidad de La Frontera y129 estudiantes corresponden a la Facultad de Educación de la Universidad Católica de Temuco. Esta muestra incluyó a 234 hombres (58.9\%) y 164 mujeres $(41.1 \%)$ con edades entre 17 y 38 años $(M=19.9, D T=1.97)$. Los estudiantes se identificaron mayoritariamente como chilenos no mapuches (84\%), con un pequeño grupo como chilenos mapuches $(13 \%)$ y un grupo residual sin identificar su origen familiar (3\%); además 18 de ellos declararon practicar algún tipo de meditación.

\section{Diseño}

Se utilizó un diseño descriptivo correlacional de grupo único, con una sola evaluación, para determinar la asociación entre Atención Plena y constructos asociados. Las variables consideradas son Atención Plena, dimensiones de personalidad, sintomatología (depresión, ansiedad, estrés), autoactualización y práctica de meditación.

\section{Instrumentos}

Five Facet Mindfulness Questionnaire (FFMQ). El cuestionario original en inglés es un autoinforme de 39 ítems que mide Atención Plena a partir de cinco facetas denominadas Observación, Descripción, Actuar con Conciencia, Ausencia de Juicio y Ausencia de Reactividad. Los participantes deben responder cada ítem en una escala Likert con un rango de 1 (nunca o muy raramente verdad) a 5 (muy a menudo o siempre verdad); la puntuación mínima del Cuestionario es de 39 puntos y la máxima de 195 puntos. De acuerdo a Baer et al. (2008) el instrumento posee adecuados a buenos niveles de confiabilidad (alfa de Cronbach de .75 a .92). En Chile, el cuestionario fue traducido al español y retraducido por psicólogos bilingües, luego fue aplicado en forma piloto a 10 personas con alta experiencia en la práctica de meditación (Cárcamo y Cruz-Coke, 2009). Posteriormente, se realizó una pre-validación del instrumento, comparando meditadores con no meditadores (Solari, 2010). Aunque en este estudio se observó buena confiabilidad, el pequeño 
tamaño de la muestra $(n=100)$ no permitió su validación. Esta versión en español es la utilizada en el presente estudio.

NEO Five-Factor Inventory (NEO-FFI). Este instrumento, diseñado por Costa y McCrae (1992), es una versión abreviada del NEO-PI. Evalúa empíricamente las cinco dimensiones de personalidad del MCF: Neuroticismo $(\mathrm{N})$, Extroversión (E), Apertura a la Experiencia (O), Agradabilidad (A) y Ser Concienzudo (C). El NEO-FFI está integrado por 60 ítems (12 ítems por cada factor) presentados como descripciones personales breves con cinco opciones de respuesta: "muy en desacuerdo", "en desacuerdo", "ni en acuerdo ni en desacuerdo", "de acuerdo", y "muy de acuerdo". El MCF cuenta en Chile con un primer estudio de validez en el cual se empleó una versión nacional del NEO-FFI (Vinet, Fuentes, Melo, Muñoz y Saiz, 1998). En este estudio se reportan adecuados niveles de confiabilidad para cada una de las cinco escalas del NEO-FFI y evidencia aceptable de validez convergente y discriminante en relación al modelo de necesidades de Murray evaluado a través de dos versiones del Inventario de Preferencias Personales de Edwards en estudiantes universitarios (ver Vinet et al., 1998).

Escalas de Depresión Ansiedad y Estrés (DASS-21). Es la versión abreviada del DASS (originalmente de 42 ítems). Es una escala de autorreporte dimensional que fue diseñada para medir los estados emocionales negativos de depresión, ansiedad y estrés. A los participantes se les solicita que respondan cada ítem de acuerdo a la presencia e intensidad de cada síntoma en la última semana. Cada una de las tres escalas tiene siete ítems con formato de respuesta tipo Likert con cuatro alternativas que se ordenan en una escala de 0 a 3 puntos. El puntaje total de cada escala varía entre 0 y 21 puntos. La versión chilena fue traducida y adaptada por Vinet, Rehbein, Román y Saiz (2008) y validada tanto en adolescentes estudiantes de enseñanza media (Román, Vinet y Alarcón, 2014) como en estudiantes universitarios (Antúnez y Vinet, 2012), observándose buenas características psicométricas en ambos estudios.

Personal Orientation Inventory (POI). Cuestionario de autorreporte desarrollado por Shostrom (1964) para evaluar la tendencia actualizante o autoactualización, según los desarrollos de las teorías humanistas. Se compone de 150 ítems donde cada ítem pertenece a una de las dos escalas generales: Autonomía (130 ítems) y Adecuación Temporal (20 ítems) y además aporta a 10 subescalas. Su formato de respuesta es de elección forzada entre dos alternativas, el sujeto elige de cada par la afirmación que a su juicio lo representa más fielmente. La adaptación y estandarización del inventario fue realizada en estudiantes universitarios de la Universidad de Chile (Celis, Farias y Iturra, 1976).
En el presente estudio se trabajó con esta versión chilena considerando las dos escalas generales.

Cuestionario Sociodemográfico. Se utilizó para registrar datos sociodemográficos y determinar la posible práctica de meditación, su tipo y su frecuencia en los participantes.

\section{Procedimiento}

El primer paso fue establecer contacto con la Dirección Académica de Pregrado de las universidades de La Frontera y Católica de Temuco. Luego de recibir autorización se coordinó con los directores de carrera y docentes la aplicación, en diferentes grupos-curso, de un cuadernillo que contenía los instrumentos ya descritos. Las instrucciones fueron iguales para todos los cursos; contestaron el cuadernillo quienes aceptaron participar luego de firmar un consentimiento informado.

\section{Análisis de datos}

Se realizó un análisis descriptivo de los resultados, determinando promedios, desviaciones estándar, medianas $\mathrm{y}$ rango de los puntajes obtenidos en cada instrumento para el total de los participantes. Posteriormente, se realizó la evaluación de la confiabilidad del FFMQ, a través del análisis de su consistencia interna (Alfa de Cronbach); estos resultados fueron comparados, mediante el estadístico W de Feldt (1969), con los índices de fiabilidad del estudio original de Baer et al. (2006). A continuación, se analizó la validez convergente y discriminante entre las medidas del FFMQ y de los instrumentos aplicados, por medio de correlaciones $r$ de Pearson, según escalas. Adicionalmente, se compararon, mediante la prueba $t$ de Student para una sola muestra, las puntuaciones en el FFMQ de los participantes que reportaron alguna experiencia en meditación u otras disciplinas orientales que buscan la meditación $(\mathrm{n}=18)$ con las puntuaciones del resto de los participantes.

\section{Resultados}

\section{Confiabilidad y relaciones entre las facetas}

Los análisis muestran que la versión nacional del FFMQ posee una adecuada consistencia interna en población chilena universitaria (Alfa total $=.79$ ); además, aunque el rango de los coeficientes es aceptable (.62 a .86), tal como 
Tabla 1. Consistencia interna del FFMQ en la muestra chilena y contrastación con estudio original.

\begin{tabular}{llcl}
\hline \multirow{2}{*}{ Facetas } & \multicolumn{2}{c}{ Alfa de Cronbach } & \\
\cline { 2 - 3 } & $\begin{array}{c}\text { FFMQ Chileno } \\
n=399\end{array}$ & $\begin{array}{c}\text { FFMQ } \\
\text { Original } \\
n=613\end{array}$ & $\begin{array}{l}W \text { de } \\
\text { Feldt }\end{array}$ \\
\hline Observar & .73 & .83 & $.629^{*}$ \\
Describir & .78 & .91 & $.409^{*}$ \\
ActCon & .84 & .87 & $.812^{*}$ \\
No Juzgar & .86 & .87 & .928 \\
No Reaccionar & .62 & .75 & $.657^{*}$ \\
\hline
\end{tabular}

Nota . ActCon $=$ Actuar con Conciencia ${ }^{*} p<.05$

Tabla 2. Correlaciones entre facetas del FFMQ

\begin{tabular}{lcccc}
\hline Facetas & Describir & ActCon & NoJuzgar & NoReaccionar \\
\hline Observar & $.13^{* *}$ & $-.11^{*}$ & $-.28^{* *}$ & $.25^{* *}$ \\
Describir & - & $<.36^{* *}$ & $.18^{* *}$ & $.15^{* *}$ \\
ActCon & - & - & $.30^{* *}$ & .07 \\
NoJuzgar & - & - & - & $-.11^{*}$ \\
\hline
\end{tabular}

Nota. ActCon $=$ Actuar con Conciencia

$* p<.05 ; * * p .01$ se observa en la Tabla 1, todos ellos son significativamente más bajos que los originales.

Para observar la estructura del instrumento, se calcularon las correlaciones bivariadas entre las cinco facetas (ver Tabla 2), siendo éstas modestas (entre .13 y .36) pero significativas en todos los casos con la excepción de Actuar con Conciencia con No Reaccionar.

\section{Validez convergente y discriminante}

Se determinaron las correlaciones $r$ de Pearson entre el FFMQ total y por facetas, con las escalas del NEO-FFI, del DASS-21 y del POI respectivamente. Los resultados se presentan en la Tabla 3 y se reportan a continuación para cada instrumento.

FFMQ con NEO-FFI. Considerando la escala total y de acuerdo a lo predicho, se observa una correlación significativa e inversa entre Atención Plena y Neuroticismo, significativa pero modesta con Apertura a la experiencia. La relación de Atención Plena con Ser Concienzudo no se predijo y mostró tener una correlación significativa y directa. Inesperado resultó la correlación con Extroversión, ya que según la literatura, no debía existir una relación entre actuar con Atención Plena y ser introvertido o extrovertido.

Tabla 3. Correlaciones del FFMQ y sus facetas con las escalas del NEO-FFI, DASS-21 y POI

\begin{tabular}{|c|c|c|c|c|c|c|}
\hline \multirow{2}{*}{ Escalas } & \multicolumn{6}{|c|}{ FFMQ } \\
\hline & Observar & Describir & ActCon & NoJuzg & NoReac & Total \\
\hline \multicolumn{7}{|l|}{ NEO-FFI } \\
\hline Neuroticismo & $.12 *$ & $-.29 * *$ & $-.41 * *$ & $-.48 * *$ & $-.14 * *$ & $-.49 * *$ \\
\hline Extroversión & .05 & $.23 * *$ & $.13^{*}$ & .08 & -.02 & $.19^{* *}$ \\
\hline Apertura & $.34 * *$ & $.13 * *$ & -.04 & .00 & $.12 *$ & $.20 * *$ \\
\hline Agradibilidad & -.02 & $.12 *$ & $.25^{* *}$ & .09 & $.10^{*}$ & $.21 * *$ \\
\hline Ser Concienzudo & .01 & $.27 * *$ & $.42 * *$ & .04 & $.14^{* *}$ & $.33 * *$ \\
\hline \multicolumn{7}{|l|}{ DASS-21 } \\
\hline Depresión & $.12 *$ & $-.16^{* *}$ & $-.31 * *$ & $-.30 * *$ & $-.16^{* *}$ & $-.32 * *$ \\
\hline Ansiedad & $.24 * *$ & -.07 & $-.23 * *$ & $-.24 * *$ & -.07 & $-.16^{* *}$ \\
\hline Estrés & $.15^{* *}$ & $-.10^{*}$ & $-.27 * *$ & $-.27 * *$ & $-.16^{* *}$ & $-.26 * *$ \\
\hline DASS Total & $.19 * *$ & $-.12 *$ & $-.31 * *$ & $-.30 * *$ & $-.15^{* *}$ & $-.28 * *$ \\
\hline \multicolumn{7}{|l|}{ POI } \\
\hline Autonomía & .07 & $.24 * *$ & .09 & $.37 * *$ & .02 & $.33^{* *}$ \\
\hline Adecuación Temporal & -.05 & $.11 *$ & $.18 * *$ & $.22 * *$ & .10 & $.22 * *$ \\
\hline
\end{tabular}

Nota . ActCon= Actuar con Conciencia; NoJuzg = No juzgar la experiencia interna; NoReac= No reaccionar ante la experiencia interna.

$* p<.05 ; * *<<.01$ 
Respecto de la relación de cada una de las facetas del FFMQ con las dimensiones del NEO-FFI, el área más robusta corresponde a la correlación inversa de Neuroticismo con las facetas Describir, Actuar con Conciencia y No Juzgar. Luego se encuentra la relación de Ser Concienzudo con las facetas Describir y Actuar con Conciencia, con una correlación significativa y positiva, y finalmente se observa una relación significativa entre Apertura a la Experiencia y Observar.

FFMQ con DASS-21. La correlación del FFMQ total con el DASS-21 total fue de $-.28(\mathrm{p}<.01)$. Diferenciado según escalas, el coeficiente de correlación de Pearson muestra una relación significativa negativa entre las escalas de Depresión, Ansiedad y Estrés del DASS-21 con las facetas Actuar con Conciencia y No Juzgar. Por otro lado, no existe correlación de la escala Ansiedad con las facetas Describir y No Reaccionar. Inesperada es la correlación positiva entre las escalas del DASS-21 y la faceta Observar, lo cual será comentado en la discusión.

FFMQ con POI. Se encontró una relación significativa y positiva entre el FFMQ y las dos escalas generales del POI. Específicamente la correlación del FFMQ es de $.33(\mathrm{p}<.01)$ con Autonomía y de $.22(\mathrm{p}<.01)$ con Adecuación Temporal. Con respecto a las facetas del FFMQ, se observó una relación significativa de la faceta No Juzgar la experiencia interna con Adecuación Temporal y Autonomía. Además, también existe una relación modesta de Describir con Autonomía y de Actuar con Conciencia con Adecuación Temporal.

Comparación de meditadores y no meditadores. Para evaluar si el FFMQ lograba diferenciar en estudiantes universitarios a personas que realizan alguna práctica meditativa (Budismo, Tai Chi, Yoga) con respecto de quienes no practican meditación, se contrastó a un pequeño grupo de meditadores $(\mathrm{n}=18)$ con respecto al grupo mayoritario de no meditadores $(\mathrm{n}=368)$ tomando a estos como el referente poblacional. La Tabla 4 muestra que el grupo de meditadores se diferencia significativamente en el puntaje total $(\mathrm{t}=$ $2.57, p<.05)$ y en la faceta Observar $(t=3.47, p<.05)$. En la faceta Describir se observó una diferencia marginalmente significativa $(p=.054)$ y en las otras facetas, la puntuación de los meditadores no difiere del grupo de referencia. Estos resultados indican que en términos generales, los meditadores son diferentes a los no meditadores, específicamente en la capacidad de Observar, que se adquiere con la práctica de técnicas meditativas y que a su vez potenciaría la capacidad de Describir la experiencia.
Tabla 4. Diferencias en el grupo meditadores $(n=18)$ respecto del referente poblacional de no meditadores $(n=368)$.

\begin{tabular}{lccc}
\hline \multirow{2}{*}{ FFMQ } & \multicolumn{3}{c}{ Meditadores } \\
\cline { 2 - 4 } & Media & $D E$ & t para una muestra \\
\hline Escala Total & 136.61 & 17.84 & $2.57^{*}$ \\
Facetas & & & \\
Observar & 31.44 & 4.20 & $3.47^{*}$ \\
Describir & 29.94 & 5.86 & 2.07 \\
ActCon & 26.00 & 5.96 & .24 \\
No Juzgar & 25.00 & 7.82 & 1.21 \\
No Reaccionar & 24.22 & 5.04 & 1.59 \\
\hline
\end{tabular}

Nota . ActCon $=$ Actuar con Conciencia ${ }^{*} \mathrm{p}<.05$

\section{Discusión}

Este estudio, orientado a verificar las características psicométricas del FFMQ en Chile, muestra que la versión nacional del instrumento posee adecuados niveles de confiabilidad para cada una de las cinco facetas y que además existe evidencia aceptable de validez convergente y discriminante en relación al MCF, con estados emocionales negativos y con la Autoactualización de las terapias humanistas; asimismo, la escala logra diferenciar entre meditadores y no meditadores.

Las facetas del FFMQ en su mayoría se relacionan entre sí, aportando de modo complementario al constructo general, manteniendo la conceptualización de la Atención Plena como un constructo multifacético, tal y como lo demostraron los autores originales. En el estudio de Baer et al. (2006), con una muestra de estudiantes norteamericanos, obtuvieron correlaciones modestas pero significativas y sólo en el caso de la faceta Observar con No Juzgar no existió relación. En el presente estudio, ambas facetas se relacionaron de manera significativa pero inversa. Lo anterior sorprende en la medida que la observación es descrita como una característica central en la Atención Plena. Sin embargo, existe el antecedente de que Baer et al. (2004) en el desarrollo del cuestionario KIMS, también reportaron una correlación significativa negativa entre Observar con No Juzgar la experiencia interna. Ellos plantearon que en individuos sin experiencia en la meditación, atender a las experiencias generalmente puede estar asociado a juzgarlas, pero que las personas con experiencia en meditación deberían mostrar mayores niveles tanto de observación como de no juzgar su experiencia. Esta tendencia ha sido 
ratificada por Baer et al. (2006) en una muestra de sujetos con experiencia en meditación donde las facetas Observar y No Juzgar correlacionaron positivamente. Por último, Baer et al. (2008) han indicado que la tendencia a notar estímulos (internos y externos) se asocia con un menor nivel de síntomas psicológicos en personas que meditan, pero no en otros; esto mostraría que la faceta Observar puede funcionar de manera diferente en los individuos con experiencia en la meditación, haciendo comprensible los resultados encontrados en la muestra chilena compuesta mayoritariamente por personas sin práctica de meditación.

Las asociaciones de las facetas de Atención Plena con otros constructos muestran relaciones diferenciadas, siendo consistentes en gran medida con las predicciones realizadas en este estudio.

En relación al MCF (Costa y McCrae, 1992), las asociaciones más robustas se presentaron con respecto a Neuroticismo y Ser concienzudo. En el primer caso, el desarrollo de la Atención Plena ayudaría a generar mayor estabilidad emocional, específicamente a través de la capacidad de describir los estados internos, ser consciente de ellos y no actuar con autocrítica. Al respecto, Individuos que informan ser más conscientes en la vida diaria son menos impulsivos (Wittmann et al. 2014). Con respecto a Ser Concienzudo, actuar con Atención Plena requiere etiquetar los estados internos y ser conscientes de la experiencia momento a momento; para ello se necesita una capacidad de planificación atenta y cuidadosa, con un autocontrol consciente, sin dejarse afectar por la emoción, aspectos que expresan la dimensión de Ser Concienzudo. Lo anterior es coherente con lo planteado por Wittmann et al. (2014), respecto a que la atención plena como rasgo se relaciona con características de personalidad relevantes para la planificación temporal diaria y la toma de decisiones. También se encontró una relación entre Apertura a la Experiencia y la faceta Observar; la cual se entiende en términos de que la curiosidad por el mundo interno y externo de un sujeto facilitaría la capacidad de "darse cuenta", así, un sujeto con apertura va a ser más capaz de aceptar lo que emerge en su conciencia. Algunos de estos resultados resultan coherentes con lo expuesto por Baer et al. (2006), quienes informaron una relación significativa de Apertura a la Experiencia con Observar (.42) y de Neuroticismo con No Juzgar (-.55) y con Actuar con Conciencia (-.44).

La relación inversa de los estados emocionales negativos de depresión, ansiedad y estrés, con las facetas Actuar con Conciencia y No Juzgar implicaría que mientras más consciente es el sujeto de lo que le ocurre y no realiza una autocrítica de ello, menos malestar psicológico experimenta.
Lo anterior resulta relevante, puesto que la práctica de Atención Plena podría servir como una estrategia para la reducción de sintomatología.

La Autoactualización se expresó a través de la relación de las escalas de Autonomía y Adecuación Temporal con las facetas Describir y No Juzgar. A este nivel es posible entender que ser autónomo; orientado hacia sí mismo e independiente del entorno, facilita la capacidad para focalizarse en describir los estados internos y asumir una postura contemplativa frente a los pensamientos y sentimientos; por el contrario, quien no goza de autonomía y depende del entorno, puede tender a enjuiciar su experiencia interna, probablemente por temor al juicio de los demás. Por otro lado, vivir centrado en el presente facilita el actuar con plena conciencia y no enjuiciar la propia experiencia. En conclusión, a un sujeto con autonomía, centrado en el aquí y el ahora, con plena conciencia del presente, le será más fácil aceptar la experiencia que surge en el momento, sin hacer un juicio de ella. Estas asociaciones reflejan la coherencia teórica entre la autoactualización u orientación personal y la Atención Plena, puesto que uno de los objetivos principales del entrenamiento en Atención Plena es acceder a un profundo estado de calma y luego centrar la atención y la conciencia de lo que está sucediendo en el propio cuerpo y la mente, es decir, en el momento presente (Droit-Volet, Fanget y Dambrun, 2015). Adicionalmente, el FFMQ logró discriminar entre personas con experiencia en meditación y quienes no la tienen, a través del puntaje total de la escala. Esto es coherente con la literatura y los hallazgos empíricos sobre la capacidad de la meditación budista para cultivarlas habilidades de la Atención Plena y la asociación entre la experiencia en meditación con las facetas de la Atención Plena (Baer et al., 2008).

Al realizar una apreciación general, se observa que la dirección de las correlaciones encontradas es casi totalmente coherente con las predicciones, con la excepción de la faceta Observar, que se relacionó positivamente con Neuroticismo y con los estados emocionales negativos de Depresión, Ansiedad y Estrés. Aún cuando la asociación es débil, éste hallazgo da cuenta de que la intención de observar los estados internos pueden generar molestia en los individuos cuando estos son negativos. Sería similar a cuando las personas reportan tener una "rumiación cognitiva", lo cual les resulta disruptivo. Frente a este efecto es importante la práctica de no juzgar y no reaccionar ante lo observado; es decir, poder aceptar una experiencia como sentirse triste, sin realizar una autocrítica y no reaccionar impulsivamente como, por ejemplo, intentar rápidamente olvidar. Los programas psicoterapéuticos que integran la 
práctica de la Atención Plena destacan la importancia de la aceptación de los síntomas y de las sensaciones físicas o emocionales desagradables, sin intentar modificarlos o evitarlos (Irarrázaval, 2010). En el programa de Terapia Cognitiva Basada en la Atención Plena para la depresión, la práctica de meditación permite adoptar una actitud de aproximación a la experiencia y frente a los patrones automáticos de rumiación, en vez de la evitación (Segal et al., 2002). De esta forma, un individuo aprende a aceptar sus reacciones emocionales automáticas y así no aumentarlas por la aversión o atracción y como consecuencia, niveles más altos de afecto positivo pueden ser experimentados (Jha et al 2010). Lo anterior es coherente con los resultados de este estudio, al encontrar una relación significativa inversa entre No juzgar la experiencia con Neuroticismo y estados emocionales negativos, y una relación modesta pero en la misma dirección entre No Reaccionar y los mismos constructos. Estos hallazgos dan cuenta de la importancia de generar aproximaciones terapéuticas que orienten a los pacientes a asumir una postura contemplativa de su experiencia y al entrenamiento de estrategias para no autocriticarse ni reaccionar impulsivamente ante los estados emocionales negativos. Como plantea Miró (2006), para poder observar la experiencia tal y como es, es necesario antes que nada aceptar la experiencia y esto es sobre todo necesario ante los estados emocionales negativos.

En síntesis, los resultados muestran que la versión nacional del FFMQ es un buen instrumento para medir la capacidad de Atención Plena en estudiantes universitarios chilenos, lo cual permite contribuir a la investigación de este constructo y posibilitar la medida de su impacto en intervenciones terapéuticas. La exploración de las facetas de Atención Plena y su relación con otras variables psicológicas resulta relevante para la comprensión de la naturaleza del constructo; la evidencia empírica sobre cada una de las facetas puede servir para la práctica clínica, como una forma de poder explicarles a los pacientes el cómo afrontar sus síntomas.

Entre las limitaciones de este estudio está la restricción de la muestra al no ser representativa de la población general, por lo tanto se recomienda seguir estudiando las propiedades psicométricas del instrumento en muestras más amplias. A futuro, teniendo en cuenta los resultados aquí expuestos, la tarea es utilizar el FFMQ como instrumento de medida de la Atención Plena tanto al desarrollar estudios que consideren el abordaje de intervenciones basadas en la Atención Plena, como al profundizar en la comprensión del constructo a través del estudio de las facetas en muestras clínicas.

\section{Referencias}

Antúnez, Z., y Vinet, E. (2012). Escalas de Depresión, Ansiedad y Estrés (DASS - 21): Validación de la Versión Abreviada en Estudiantes Universitarios Chilenos. Terapia Psicológica, 30, 49-55.

Baer, R., Smith, G., y Allen, K. (2004). Assessment of mindfulness by self-report: The Kentucky Inventory of Mindfulness Skills. Assessment, 11, 191-206.

Baer, R., Smith, G., Hopkins, J., Krietemeyer, J., y Toney, L. (2006). Using self-report assessment methods to explore facets of mindfulness. Assessment, 13, 27-45.

Baer, R., Smith, G., Lykins, E., Button, D., Krietemeyer, J., Sauer, S., Walsh, E., Duggan, D., y Williams, J. (2008). Construct validity of the Five Facet Mindfulness Questionnaire in meditating and nonmeditating samples. Assessment, 15, 329-342.

Bishop S. R., Lau M., Shapiro S., Carlson L., Anderson N. D., Carmody J., Segal Z. V., Abbey S., Speca M., Velting D., y Devins G. (2004). Mindfulness: A proposed operational definition. Clinical Psychology: Science and Practice, 11, 230-241.

Brown, K., y Ryan, R. (2003). The benefits of being present: Mindfulness and its role in psychological well-being. Journal of Personality and Social Psychology, 84, 822-848.

Buchheld, N., Grossman, P., y Walach, H. (2001). Measuring mindfulness in insight meditation (Vipassana) and meditation-based psychotherapy: The development of the Freiburg Mindfulness Inventory (FMI). Journal for Meditation and Meditation Research, 1, 11-34.

Cahn, B. R., y Polich, J. (2009). Meditation states and traits: EEG, ERP, and neuroimaging studies. Psychological Bulletin, 132, 180-211.

Cárcamo, M., y Cruz-Coke, M. (2009). Traducción autorizada del FFMQ por autora en contexto proyecto FONIS SA 09I20014, Santiago, Chile.

Carlson, L., y Brown, K. (2005). Validation of the mindful attention awareness scale in a cancer population. Journal of Psychosomatic Research 58, 21-33.

Celis, A., Farias, R., y Iturra, A. (1976). Estandarización del inventario de actualización personal (P.O.I) en una muestra de alumnos de la Universidad de Chile. (Tesis de título profesional no publicada). Universidad de Chile, Santiago, Chile.

Chadwick, P., Hember, M., Mead, S., Lilley, B., y Dagnan, D. (2005). Responding mindfully to unpleasant thoughts and images: Reliability and validity of the Mindfulness Questionnaire. Unpublished manuscript.

Chambers, R., Lo, B. C. Y., y Allen, N. B. (2008). The impact of intensive mindfulness training on attentional control, cognitive style, and affect. Cognitive Therapy and Research, 32, 303-322.

Costa, P. T., y McCrae, R. R. (1992). Revised NEO Personality Inventory (NEO PI-R) and NEO Five-Factor Inventory (NEO-FFI): Professional manual. Odessa, FL: Psychological Assessment Resources.

Droit-Volet. S., Fanget, M., y Dambrun, M. (2015). Mindfulness meditation and relaxation training increases time sensitivity. Consciousness and Cognition, 31, 86-97.

Feldman, G. C., Hayes, A. M., Kumar, S. M., y Greeson, J. M. (2004). Development, factor structure, and initial validation of the Cognitive and Affective Mindfulness Scale. Unpublished manuscript.

Feldt, L. S. (1969). A test of the hypothesis that Cronbach's alpha or Kuder-Richardson coefficient twenty is the same for two tests. Psychometrika, 34, 363-373.

Hoffman, S. G., Sawyer, A. T., Witt, A. A., y Oh, D. (2010). The effect of mindfulness-based therapy on anxiety and depression: A meta-analytic review. Journal of Consulting and Clinical Psychology, 78, 169-183.

Irarrázaval, L. (2010). Psicoterapia basada en mindfulness: Un antiguo método para una nueva psicoterapia.Gaceta de Psiquiatría Universitaria, 6, 465-471.

Jankowski, T., y Holas, P. (2014). Metacognitive model of mindfulness. Consciousness and Cognition, 28, 64-80.

Jha, A. P., Stanley, E. A., Kiyonaga, A., Wong, L., \& Gelfand, L. (2010). Examining the protective effects of mindfulness training on working memory capacity and affective experience. Emotion, 10, 54-64. 
Kabat-Zinn, J. (1982). An outpatient program in behavioral medicine for chronic pain patients based on the practice of mindfulness meditation: Theoretical considerations and preliminary results. General Hospital Psychiatry, 4, 33-47.

Kabat-Zinn, J. (1990). Full catastrophe living: Using the wisdom of your mind and body to face stress, pain, and illness. NewYork: Delacorte.

Kabat-Zinn, J. (2003). Minfulness-based interventions in context: past, present and future. Clinical Psychology: Science and Practice. 10, 144-156.

Linehan, M. M. (1993a). Cognitive-behavioral treatment of borderline personality disorder. New York: Guilford.

Linehan, M. M. (1993b). Skills training manual for treating borderline personality disorder. New York: Guilford.

Medeiros, S. y Pulido, R. (2011) Programa de Reducción de Estrés basado en Mindfulness para funcionarios de la salud: experiencia piloto en un hospital público de Santiago de Chile. Revista Chilena de Neuro Psiquiatría, 49, 251-257.

Miró, M. T. (2006). La Atención Plena (Mindfulness) como intervención clínica para aliviar el sufrimiento y mejorar la convivencia, Revista de Psicoterapia, 17 (66-67), 31-76.

Román, F., Vinet, E. V., \& Alarcón, A. M. (2014) Escalas de Depresión Ansiedad Estrés (DASS-21): Adaptación y Propiedades Psicométricas en estudiantes secundarios de Temuco. Revista Argentina de Clínica Psicológica, 23(2), 179-190.

Segal, Z. V., Williams, J. M. G., y Teasdale, J. D. (2002). Mindfulness based cognitive therapy for depression: A new approach to preventing relapse. New York: Guilford.

Shostrom, E. (1964). An inventory for the measurement of self-actualization. Educational and Psychological Measurement, 24, 207-218.

Shostrom, E. (1977) Manual for the Personal Orientation Dimensions. Edits, San Diego, California.

Solari, B. (2010). Pre validación del cuestionario five facet mindfulness questionnaire. (Tesis de Magíster no publicada). Pontificia Universidad Católica de Chile, Santiago, Chile.

Vallejo, M. A. (2006). Mindfulness. Papeles del Psicólogo, 27, 92-99.

Vinet, E., Fuentes, M., Melo, G., Muñoz, R., y Saiz, J. L. (1998). Necesidades y Rasgos: una réplica transcultural del estudio de Piedmont, McCrae y Costa (1992). Revista de Psicología Social y Personalidad, 14, 29-40.

Vinet, E., Rehbein, L., Román F., y Saiz, J. (2008). Escalas abreviadas de depresión, ansiedad y estrés (dass-21). Versión chilena traducida y adaptada. (Documento no publicado). Universidad de La Frontera, Temuco, Chile.

Wittmann, M., Peter. J., Gutina, O., Otten, S., Kohls, N., Meissner, K. (2014). Individual differences in self-attributed mindfulness levels are related to the experience of time and cognitive self-control. Personality and Individual Differences, 64, 41-45. 
